\section{Chamada para ação emergencial para limitar - aumento da temperatura global, restaurar a biodiversidade e proteger a saúde}

Lukoye Atwoli 1 Abdullah H. Baqui 2

Thomas Benfield 3 Raffaella Bosurgi 4 Fiona Godlee 5 Stephen Hancocks 6 Richard Horton 7

Laurie LaybournLangton 8

Carlos Augusto

Monteiro 9

Ian Norman 10
Kirsten Patrick 11

Nigel Praities 12

Marcel G. M. Olde

Rikkert 13

Eric J. Rubin 14

Peush Sahni 15

Richard Smith 16

Nick Talley 17

Sue Turale 18

Damián Vázquez 19

doi: 10.1590/0102-311X00194721

\section{Os países ricos devem fazer muito mais, e muito mais rapidamente}

Em setembro de 2021, a Assembleia Geral das Nações Unidas reunirá os países em um momento crítico para mobilizar a ação coletiva no enfrentamento da crise ambiental global. As nações estarão reunidas novamente na Cúpula da Biodiversidade em Kunming, na China, e na Conferência das Partes sobre Clima (COP26) em Glasgow, Reino Unido. Antes desses encontros cruciais, nós - editoras e editores de revistas de saúde do mundo inteiro - lançamos esta chamada para ação urgente a fim de manter o aumento médio da temperatura global abaixo de $1,5^{\circ} \mathrm{C}$, interromper a destruição da natureza e proteger a saúde.

A saúde já está sendo prejudicada pelo aumento da temperatura global e pela destruição do mundo natural, situação essa que os profissionais de saúde vêm denunciando há décadas 1 . A ciência é inequívoca; um aumento global de $1.5^{\circ} \mathrm{C}$ acima da média pré-industrial e a continuação da perda da biodiversidade significam risco de danos irreversíveis à saúde 2,3. Apesar da preocupação necessária do mundo em relação à COVID-19, não podemos esperar o fim da pandemia para reduzir rapidamente as emissões.

Como reflexo da gravidade do momento, este editorial aparece em revistas de saúde do mundo inteiro. Estamos unidos no reconhecimento de que a trajetória atual somente será revertida através de mudanças fundamentais e equitativas em nossas sociedades.

Já estão bem documentados os riscos para a saúde, ocasionados por aumentos de temperatura acima de $1,5^{\circ} \mathrm{C} 2$. Em realidade, nenhum aumento de temperatura é "seguro". Nos últimos 20 anos, a mortalidade relacionada ao calor entre pessoas acima de 65 anos aumentou em mais de $50 \% 4$. Temperaturas elevadas têm resultado em aumento de desidratação e perda de função renal, neoplasias dermatológicas, infecções tropicais, transtornos mentais, complicações na gravidez, alergias e morbimortalidade cardiovascular e pulmonar 5,6. Os danos afetam de maneira desproporcional os mais vulneráveis, incluindo crianças, idosos, minorias étnicas, comunidades pobres e indivíduos com comorbidades 2,4

O aquecimento global também está contribuindo com o declínio da produtividade potencial global dos principais cultivos agrícolas, que sofreram quedas entre 1,8\% e 5,6\% desde 1981; junto com os efeitos climáticos extremos e o esgotamento do solo, o aquecimento está dificultando os esforços para reduzir a desnutrição ${ }^{4}$. Ecossistemas pujantes são essenciais para a saúde humana, e a destruição desenfreada da natureza, incluindo habitats e espécies, está minando a segurança hídrica e alimentar e aumentando o risco de pandemias $3,7,8$.

As consequências da crise ambiental recaem de maneira desproporcional sobre países e comunidades que contribuíram menos para o problema e que têm menores condições de mitigar os danos.
1 East African Medical Journal, Nairobi, Kenya. 2 Journal of Health, Population and Nutrition, Dhaka, Bangladesh. 3 Danish Medical Journal, Copenhagen, Denmark. 4 PLoS Medicine, San Francisco, U.S.A.

5 The British Medical Journal, London, England. ${ }_{6}^{6}$ British Dental Journal, London, England.

7 The Lancet, London, England.

8 UK Health Alliance on Climate Change, London, England.

${ }^{9}$ Revista de Saúde Pública, São Paulo, Brasil. 10 International Journal of Nursing Studies, Oxford, England.

11 Canadian Medical Association Journal, Ottawa, Canada.

12 Pharmaceutical Journal, London, England.

${ }_{13}$ Dutch Journal of Medicine, Amsterdan, Netherlands. ${ }_{14}$ New England Journal of Medicine, Boston, U.S.A. 15 National Medical Journal of India, New Delhi, India. ${ }_{16}$ UK Health Alliance on Climate Change, London, England.

17 Medical Journal of Australia, Strawberry Hills, Australia.

18 International Nursing Review, Oxford, England. 19 Pan American Journal of Public Health, Washington D.C., U.S.A. 
No entanto, nenhum país, por mais rico que seja, consegue se resguardar desses impactos. Permitir que as consequências prejudiquem de maneira desproporcional os mais vulneráveis levará a mais conflitos, insegurança alimentar, deslocamento forçado e zoonoses - com implicações graves para todos os países e comunidades. Assim como na pandemia da COVID-19, em nível global somos apenas tão fortes quanto os membros mais fracos.

Elevações térmicas acima de $1,5^{\circ} \mathrm{C}$ aumentam o risco de chegar ao ponto sem volta nos sistemas naturais, deixando o mundo preso em um estado agudamente instável. Isso comprometeria de maneira crítica nossa capacidade de mitigar os danos e de prevenir a mudança climática desenfreada e catastrófica 9,10 .

\section{As metas globais não bastam}

É animador que muitos governos, instituições financeiras e empresas estejam definindo metas para alcançar a emissão líquida zero, incluindo metas para 2030. O custo da energia renovável está caindo rapidamente. Muitos países pretendem proteger pelo menos 30\% das terras e oceanos até 203011.

Mas as promessas não bastam. As metas são fáceis de definir e difíceis de atingir. Ainda falta combiná-las com planos no curto e longo prazo para acelerar as tecnologias mais limpas e transformar as sociedades. Os planos de redução de emissões não incorporam adequadamente as questões de saúde 12 . Há uma preocupação crescente de que os aumentos de temperatura acima de $1,5^{\circ} \mathrm{C}$ estejam começando a ser vistos como inevitáveis, ou até aceitáveis, por membros poderosos da comunidade global 13. De maneira correlata, as estratégias atuais de redução até chegar à emissão líquida zero em meados do século XXI pressupõem, de maneira implausível, que o mundo possa adquirir a capacidade monumental de remover os gases de efeito estufa da atmosfera 14,15 .

Essa ação insuficiente significa que as elevações de temperatura deverão superar de longe $2^{\circ} \mathrm{C} 16$, um cenário catastrófico para a estabilidade sanitária e ambiental. Uma questão crítica é que a destruição da natureza não parece receber a mesma atenção dada ao componente propriamente climático da crise, na medida em que, para a restauração da biodiversidade até 2020, nenhuma das metas foi atingida 17 . E isso é uma crise ambiental generalizada 18.

Os profissionais da saúde estão unidos aos cientistas ambientais, empresas e muitos outros na recusa da inevitabilidade desse desfecho. É possível e imperativo fazer mais agora - em Glasgow e Kunming - e nos anos imediatamente seguintes. Solidarizamo-nos com os profissionais da saúde do mundo inteiro que já vêm apoiando as chamadas para ação rápida 1,19.

A equidade deve estar no centro da resposta global. A contribuição para uma parcela justa do esforço global significa que os compromissos para a redução das emissões devem levar em conta a contribuição histórica cumulativa que cada país fez às emissões, assim como, suas emissões atuais e sua capacidade de responder. Os países mais ricos terão que cortar as emissões mais rapidamente, alcançando reduções até 2030 além daquelas propostas atualmente 20,21 e atingindo emissões zero antes de 2050. São necessárias metas semelhantes e ação emergencial em relação à perda da biodiversidade e à destruição geral do mundo natural.

Para alcançar essas metas, os governos devem implementar mudanças fundamentais na organização de nossas sociedades e economias e na maneira que nós vivemos. Não basta a estratégia atual de incentivar os mercados a trocarem as tecnologias sujas por outras mais limpas. Os governos devem intervir para redesenhar os sistemas de transporte, as cidades, a produção e distribuição de alimentos, os mercados de investimentos financeiros, os sistemas de saúde e muito mais. A coordenação global é necessária para assegurar que a corrida para as tecnologias mais limpas não venha acompanhada por mais destruição ambiental e exploração humana.

Muitos governos responderam à ameaça da pandemia da COVID-19 com financiamento sem precedentes. A crise ambiental exige uma resposta emergencial semelhante. Será necessário um investimento gigantesco, para além daquele que está sendo considerado ou realizado em qualquer lugar 
do mundo. Entretanto, esses investimentos produzirão benefícios sanitários e econômicos positivos enormes, que incluem: empregos de alta qualidade, redução da poluição atmosférica, aumento da atividade física e melhora da moradia e da dieta. A melhor qualidade do ar por si só resultaria em benefícios para a saúde que poderiam facilmente compensar os custos globais da redução das emissões 22 .

Essas medidas também irão melhorar os determinantes sociais e econômicos da saúde, o estado precário dos quais pode ter deixado as populações mais vulneráveis à pandemia da COVID-19 23. Mas as mudanças não poderão ser realizadas através de uma volta às deletérias políticas de austeridade ou pela persistência das grandes desigualdades de riqueza e poder dentro e entre países.

\section{A cooperação exige que as nações ricas façam mais}

Especificamente, os países que têm criado de maneira desproporcional a crise ambiental devem fazer mais para apoiar os países de renda baixa e média no sentido de construir sociedades mais limpas, mas saudáveis e mais resilientes. Os países de renda alta devem atingir e superar o compromisso assumido por eles, de prover USD 100 bilhões por ano, compensando em 2020 qualquer falta em anos anteriores e aumentando as contribuições até 2025 e além. O financiamento deve ser dividido igualmente entre a mitigação e a adaptação, incluindo a melhora da resiliência dos sistemas de saúde.

O financiamento deve feito na forma de fundo perdido em vez de empréstimos, construindo as capacidades locais e empoderando verdadeiramente as comunidades, e deve ser acompanhado do perdão das dívidas grandes, que limitam a autonomia de tantos países de renda baixa. Deve ser mobilizado financiamento adicional para compensar as perdas e danos inevitáveis provocados pelas consequências da crise ambiental.

Enquanto profissionais da saúde, devemos fazer tudo que estiver ao nosso alcance para ajudar a transição para um mundo sustentável, mais justo, mais resiliente e mais saudável. Junto com a ação para reduzir os danos da crise ambiental, devemos contribuir proativamente para a prevenção global de mais danos, e para mais ação sobre as causas básicas da crise. Devemos cobrar dos líderes globais e continuar educando outros sobre os riscos para a saúde provenientes da crise. Devemos nos juntar no trabalho de construir sistemas de saúde ambientalmente sustentáveis antes de 2040, reconhecendo que isso significará mudanças na prática clínica. As instituições de saúde já desinvestiram mais de USD 42 bilhões de ativos em combustíveis fósseis; outros setores devem seguir nessa mesma linha 4.

A maior ameaça à saúde pública global é o fracasso dos líderes mundiais no sentido de manter a elevação da temperatura global abaixo de $1,5^{\circ} \mathrm{C}$ e de restaurar a natureza. Mudanças urgentes devem ser feitas, abrangendo toda a sociedade; tais mudanças levarão a um mundo mais justo e mais saudável. Nós, enquanto editoras e editores de revistas de saúde, conclamamos aos governos e às outras lideranças para agirem, consagrando 2021 como o ano em que o mundo finalmente muda de rumo. 


\section{Informações adicionais}

ORCID: Lukoye Atwoli (0000-0001-7710-9723); Abdullah H. Baqui (0000-0001-8350-1983); Thomas Benfield (0000-0003-0698-9385); Raffaella Bosurgi (0000-0001-7526-6514); Fiona Godlee (0000-0002-9681-285X); Stephen Hancocks (0000-0002-5328-8509); Richard Horton (00000003-1792-5408); Laurie Laybourn-Langton (0000-0002-6605-0794); Carlos Augusto Monteiro (0000-0002-3777-1533); Ian Norman (0000-00017567-2977); Kirsten Patrick (0000-0003-45258323); Nigel Praities (0000-0002-8936-8471); Marcel G. M. Olde Rikkert (0000-0003-1397-1677); Eric J. Rubin (0000-0001-5120-962X); Peush Sahni (0000-0002-6910-062X); Richard Smith (00000001-9634-2918); Nick Talley (0000-0003-25373092); Sue Turale (0000-0001-8704-5161); Damián Vázquez (0000-0003-3551-9251).

1. Healthy Recovery. In support of a health recovery. https://healthyrecovery.net.

2. Intergovernmental Panel on Climate Change. Summary for policymakers. In: Global warming of $1.5^{\circ} \mathrm{C}$. An IPCC special report on the impacts of global warming of $1.5^{\circ} \mathrm{C}$ above pre-industrial levels and related global greenhouse gas emission pathways, in the context of strengthening the global response to the threat of climate change, sustainable development, and efforts to eradicate poverty. https://www. ipcc.ch/sr15/.

3. Intergovernmental Science-Policy Platform on Biodiversity and Ecosystem Services. Summary for policymakers: the global assessment report on biodiversity and ecosystem services. 2019. https://ipbes.net/sites/default/files/2020-02/ ipbes_global_assessment_report_summary_ for_policymakers_en.pdf.

4. Watts N, Amann M, Arnell N, Ayeb-Karlsson S, Beagley J, Belesova K, et al. The 2020 report of the Lancet Countdown on health and climate change: responding to converging crises. Lancet 2021; 397:129-70.

\section{Conflito de interesses}

F. Godlee é membro do comitê executivo da Aliança da Saúde sobre Mudança Climática do Reino Unido e do conselho de administradores do Eden Project. R. Smith é presidente da entidade Patients Know Best, possui ações na UnitedHealth Group, prestou consultoria para a Oxford Pharmagenesis e é presidente da Comissão Lancet sobre o Valor da Morte. Nenhum outro potencial conflito a declarar.

\section{Fonte e revisão de pares}

Texto encomendado; sem revisão de pares.

Este editorial está sendo publicado simultaneamente em muitas revistas científicas internacionais. Favor consultar a lista completa em: https://www. bmj.com/content/full-list-authors-and-signatoriesclimate-emergency-editorial-september-2021.

5. Rocque RJ, Beaudoin C, Ndjaboue R, Cameron L, Poirier-Bergeron L, Poulin-Rheault R-A, et al. Health effects of climate change: an overview of systematic reviews. BMJ Open 2021; 11:e046333.

6. Haines A, Ebi K. The imperative for climate action to protect health. N Engl J Med 2019; 380:263-73.

7. United Nations Environment Programme and International Livestock Research Institute. Preventing the next pandemic: zoonotic diseases and how to break the chain of transmission. https://72d37324-5089-459c-8f70$271 \mathrm{~d} 19427$ cf2.filesusr.com/ugd/056cf4_ b5b2fc067f094dd3b2250cda15c47acd.pdf.

8. Arnet A, Brabosa H, Benton TG, Calvin K, Calvo E, Connors S, et al. Summary for policymakers. In: Climate change and land: an IPCC special report on climate change, desertification, land degradation, sustainable land management, food security, and greenhouse gas fluxes in terrestrial ecosystems. https://www.ipcc.ch/ $\mathrm{srccl} /$ chapter/summary-for-policymakers/. 
9. Lenton TM, Rockström J, Gaffney O, Rahmstorf S, Richardson K, Steffen W, et al. Climate tipping points - too risky to bet against. Nature 2019; 575:592-5.

10. Wunderling N, Donges JF, Kurths J, Winkelmann R. Interacting tipping elements increase risk of climate domino effects under global warming. Earth System Dynamics 2021; 12:601-19.

11. High Ambition Coalition. https://www.hac fornatureandpeople.org.

12. Global Climate and Health Alliance. Are national climate commitments enough to protect our health? https://climateandhealthalliance. org/initiatives/healthy-ndcs/ndc-scorecards/.

13. Climate strikers: open letter to EU leaders on why their new climate law is 'surrender.' Carbon Brief 2020. https://www.carbonbrief.org/ climate-strikers-open-letter-to-eu-leaderson-why-their-new-climate-law-is-surrender.

14. Fajardy M, Köberle A, MacDowell N, Fantuzzi A. "BECCS deployment: a reality check." Grantham Institute briefing paper 28, 2019. https://www.imperial.ac.uk/media/imperialcollege/grantham-institute/public/publica tions/briefing-papers/BECCS-deployment--a-reality-check.pdf.

15. Anderson K, Peters G. The trouble with negative emissions. Science 2016; 354:182-3.

16. Climate Action Tracker. https://climateaction tracker.org.

17. Secretariat of the Convention on Biological Diversity. Global biodiversity outlook 5 . https://www.cbd.int/gbo5.
18. Steffen W, Richardson K, Rockström J, Cornell SE, Fetzer I, Bennett EM, et al. Sustainability. Planetary boundaries: guiding human development on a changing planet. Science 2015; 347:1259855.

19. UK Health Alliance. Our calls for action. http://www.ukhealthalliance.org/cop26/.

20. Climate Action Tracker. Warming projections global update: may 2021. https://climateac tiontracker.org/documents/853/CAT_202105-04_Briefing_Global-Update_ClimateSummit-Momentum.pdf.

21. United Nations Environment Programme. Emissions gap report 2020. Nairobi: United Nations Environment Programme; 2020.

22. Markandya A, Sampedro J, Smith SJ, Van Dingenen R, Pizarro-Irizar C, Arto I, et al. Health co-benefits from air pollution and mitigation costs of the Paris Agreement: a modelling study. Lancet Planet Health 2018; 2:e126-33.

23. Paremoer L, Nandi S, Serag H, Baum F. Covid-19 pandemic and the social determinants of health. BMJ 2021; 372:n129.

Recebido em 04/Ago/2021

Aprovado em 09/Ago/2021 